\title{
Numerical Quadrature of Fourier Transform Integrals II
}

\author{
By H. Hurwitz, Jr., R. A. Pfeiffer and P. F. Zweifel*
}

1. Introduction. In a previous paper [1], even-point Gaussian integration formulae for the numerical quadrature of the trigonometric integrals

$$
S(x)=\int_{0}^{\infty} \phi(k) \sin k x d k
$$

and

$$
C(x)=\int_{0}^{\infty} \psi(k) \cos k x d k
$$

were presented. In this note we present odd-point Gaussian formulae. In addition. by making use of the fact that in both the even and odd point schemes the points are equally spaced, we derive a method for evaluating the integrals in Eq. (1) for a large number of values of the parameter $x$ from a knowledge of the functions $\phi(k)$ or $\psi(k)$ at a specified set of points $\left\{k_{i}\right\}$. In other words, we remove, to some extent, one of the disadvantages of the method described in [1], to wit, that for each different value of $x$ the functions $\psi$ and $\phi$ had to be evaluated at an entirely new set $\left\{k_{i}\right\}$.

This disadvantage is also inherent in a scheme proposed by Goldberg and Varga [2], whose method also appears to be somewhat more difficult to apply than that used here.

2. Odd Point Gaussian Formula. In [1] the integral (1a) was transformed into the following form (the arguments for (1b) are identical):

$$
S(x)=\frac{\pi}{2 x} \int_{-\frac{1}{2}}^{\frac{1}{2}} \cos \pi y \sigma(x, y) d y
$$

with

$$
\sigma(y, x)=\sum_{n=-\infty}^{\infty}(-)^{n} \phi\left(\frac{\pi}{x}\left[y+n+\frac{1}{2}\right]\right)
$$

The ( $2 N$ point) Gaussian formula gives for the integral

$$
S(x)=\frac{\pi}{2} \sum_{j=1}^{N} \frac{2 W_{j}^{(N)}}{\cos \pi y_{j}^{(N)}} \sigma\left(y_{j}^{(N)}, x\right)
$$

where the $y_{j}^{(N)}$ are roots of the equation

$$
\frac{T_{2 N+1}(\cos \pi y)}{\cos \pi y}=0 .
$$

Received June 12, 1958.

* Present affiliation: Department of Nuclear Engineering, University of Michigan, Ann Arbor, Michigan. 
Here $T_{2 N+1}$ is a Chebyshev polynomial of the first kind [3]. The $W_{j}$ are the Christoffel numbers which may be obtained from Eq. (14) of [1] which was derived from the fact that the Gaussian formula gives a rigorous answer if $\sigma(y, x) / \cos \pi y$ is any polynomial of degree up to and including $N-1$ in $\cos ^{2} \pi y$.

The $2 N+1$ point formula $N=1,2,3 \cdots$ is similar to Eq. (1), except that the $y_{j}{ }^{(N)}$ are the roots of the equation

$$
U_{2 N+1}(\sin \pi y)=0
$$

where $U_{n}$ is a Chebyshev polynomial of the second kind [3],

$$
U_{n}(\cos \pi y)=\frac{\sin (n+1) \pi y}{\sin \pi y} .
$$

Then, the quadrature formula is simply

$$
S(x)=\frac{\pi}{2 x}\left\{W_{0}^{(N)} \sigma(0, x)+\sum_{j=1}^{N} \frac{2 W_{j}^{(N)}}{\cos \pi y_{j}^{(N)}} \sigma\left(y_{j}^{(N)}, x\right)\right\}
$$

where the first term of (8) has been split off since Eq. (6) has a root at $y=0$ for all $N$.

The Christoffel numbers are found from the following $(N+1)$ simultaneous equations:

$$
\frac{1}{\sqrt{ } \pi} \frac{\Gamma\left(\lambda+\frac{1}{2}\right)}{\Gamma(\lambda+1)}=\left\{W_{0}^{(N)}+2 \sum_{j=1}^{N} \cos ^{2 \lambda-2} y_{j}^{N} W_{j}^{(N)}\right\} .
$$

The $y_{j}^{(N)}$ (for the $2 N+1$ point formula) are

$$
y_{j}^{(N)}=\frac{j}{2 N+2}, \quad j=1,2,3, \cdots N .
$$

Fig. 1 of reference [1] showing the error in percent in the 1, 2, and 4 point formulae, is reproduced as Fig. 1 of this paper, with additional curves showing the errors in the 3 and 5 point formulae. The summation procedures are, of course, identical for odd- and even-point formulae.

3. Integration Over a Fixed Point Set $\left\{k_{i}\right\}$. In many applications, it may be necessary to evaluate the integrals of Eqs. (1) for a large number of values of the parameter $x$. In general, for each $x$, the transform function must be evaluated at a different set $\left\{k_{i}\right\}$. However, by taking advantage of the fact that the points of both the odd and even point Gaussian quadrature schemes are equally spaced, it is possible to choose initially a set $\left\{k_{i}\right\}$ at which the function $\phi(k)$ is to be evaluated, and these points are used in the integration scheme for each value of $x$. Clearly, $S(x)$ cannot be obtained for all $x$; one initially chooses a value $x_{\max }$ which is the maximum value of the argument for which one wishes to evaluate the function $S(x)$. Then it is possible to find $S\left(x_{\max } / \frac{1}{2} m\right)$, from the $\phi\left(k_{i}\right), m=2,3,4 \cdots,{ }^{*}$ which in practice, will frequently be enough points to allow a smooth curve of the functions $S(x)$ to be drawn.

* For the cosine integral, $m=1$ is also a permissible value. Thus, $x_{\max }$ may be chosen as one-half the maximum desired value of $x$ for which the cosine integral is desired. 


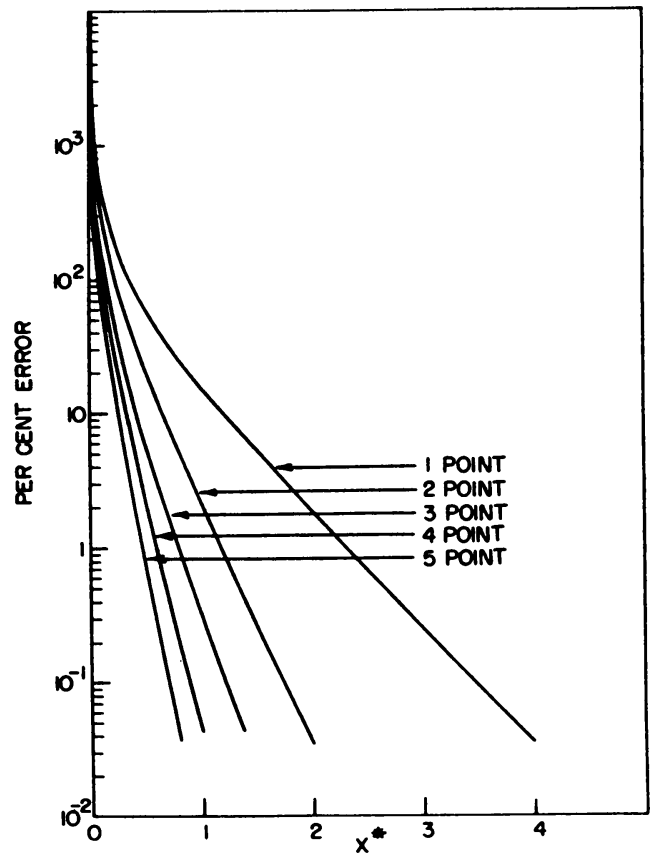

Fig. 1.-The per cent error introduced by evaluating the integral $C(x)$ by the one, two, three, four and five point formulas, with $\psi(k)=\left(1+L^{2} k^{2}\right)^{-1}$ plotted as a function of $x^{*} ; x^{*}=$ $x / L$.

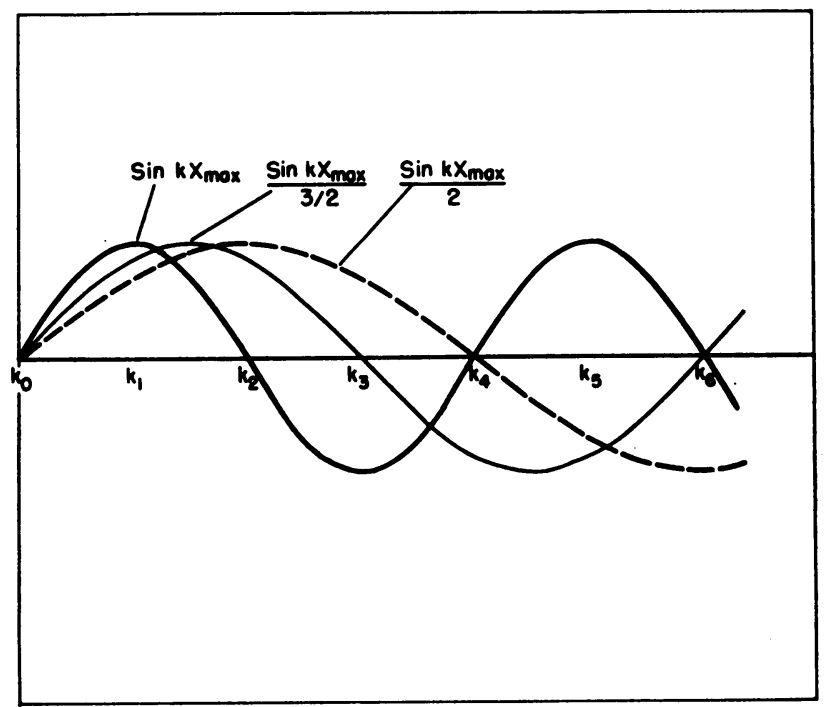

Fig. 2.-Sin $k x_{\max }, \sin k x_{\max } / \frac{8}{8}$ and $\sin k x_{\max } / 2$ vs $k$.

The required points $k_{i}$ are

$$
k_{i}=\frac{i \pi}{2 x_{\max }}, \quad \quad i=0,1,2 \cdots .
$$

Note that this is twice as many points as are needed to evaluate $S\left(x_{\max }\right)$; the 
additional points are used for evaluation of the smaller values of $x$. For each such value, some of the points will be "wasted", since the $k_{i}$ are chosen such that the zeros of $\sin (k x)$ always fall at one of the $k_{i}$ for the values of $x$ that we consider. We make this choice because it appears to be the most convenient for our purposes. It means that for every $n, S\left(x_{\max } /(n / 2)\right)$ can be evaluated with equi-spaced points, the points falling at the zeros of $\sin k x_{\max } /(n / 2)$ being weighted with a Christoffel number $W_{j}=0$.

Fig. 2 illustrates the points $\left\{k_{i}\right\}$ at which $\phi(k)$ is evaluated, and the possible values of $x$ which may be found from these $\left\{k_{i}\right\}$.

It is clear, from the figure, that the integral involving sin $k x_{\max }$ can be evaluated from a 1 point formula, that involving $\left(\sin k x_{\max }\right) /(3 / 2)$ from a 2 point formula, that involving $\left(\sin k x_{\max }\right) / 2$ from a 3 point or a one point formula, etc. If convenient, for machine calculational purposes, the end points can be included, so that an $N$ point formula becomes an $N+2$ point formula with the Christoffel numbers of the first and last points of each half cycle being set equal to zero. This, as was mentioned previously, makes the point spacing equidistant everywhere.

Consider $S\left(x_{\max } /(n / 2)\right)$. Clearly, not every $N$ point Gaussian formula involving the points $\left\{k_{i}\right\}$ can be used. We find the available formulas in the following manner:

We write all the factors of $n$ including $n$ but not 1 (not only prime factors).

Then, if these factors are $f_{1}, f_{2}, f_{j}$, the available formulas are $\left(f_{1}-1\right)$ point, $\left(f_{2}-1\right)$ point, $\left(f_{j}-1\right)$ point. (Note that when $n$ is prime only one and $(n-1)$ point formulae may be used.)

For an $\left(f_{i}-1\right)$ point formula, $\phi(k)$ is evaluated at the points $k_{\alpha}$, where $\alpha=\frac{j n}{f_{i}} \quad j=\underset{\text { first half cycle }}{\left(0,1,2, \cdots, f_{i}\right) ;} \quad \begin{array}{cc}\left(f_{i}, f_{i}+1, \cdots 2 f_{i}\right) ; & \left(2 f_{i}, 2 f_{i}+1, \cdots 3 f_{i}\right) . \\ \text { second half cycle } & \text { third half cycle }\end{array}$

The Christoffel numbers associated with the points corresponding to $j=2, \cdots$ $f_{i}-1 ; f_{i}+1, \cdots 2 f_{i}-1$; etc. are those obtained for the $\left(f_{i}-1\right)$ point formula, while the end points $h f_{i}, h=0,1, \cdots$ have Christoffel numbers zero in order to simplify machine coding.

General Electric Research Laboratory,

Schenectady, New York and

Knolls Atomic Power Laboratory,

Schenectady, New York

1. H. HURwitz, JR. \& P. F. ZwEIFEL, "Numerical quadrature of Fourier transform integrals," No. 55, MTAC, v. X, 1956, p. 140-149.

2. R. R. GoldBerg \& R. S. VARGA, "Moebius inversion of Fourier transforms," AEC Report, WAPD-TN-524, 1956.

3. A. Erdelyi, W. Magnus, F. Oberhettinger \& F. G. Tricomi, Higher Transcendental Functions, v. 2, McGraw Hill, Inc., New York, 1953. 